\title{
Postoperative C-reactive protein/albumin ratio as a novel predictor for short-term complications following gastrectomy of gastric cancer
}

\author{
Feng Sun ${ }^{1}$, Xiaolong Ge ${ }^{2}$, Zhijian Liu', Shangce Du ${ }^{1}$, Shichao $\mathrm{Ai}^{1}$ and Wenxian Guan ${ }^{1 *}$
}

\begin{abstract}
Background: Postoperative complications following gastric cancer resection remain a clinical problem. Early detection of postoperative complications is needed before critical illness develops. The purpose of this study was to evaluate the prognostic value of $\mathrm{C}$-reactive protein/albumin ratio in patients with gastric cancer.

Methods: A total of 322 patients undergoing curative (R0) gastrectomy between 2015 and 2017 were retrospectively analyzed. Univariate and multivariate analyses were performed to identify clinical factors predicting postoperative complications. The cutoff values and diagnostic accuracy of C-reactive protein/albumin ratio and C-reactive protein were determined by receiver-operating characteristic curves.

Results: Among all of the patients, 85 (26.4\%) developed postoperative complications. The optimal cutoff of C-reactive protein/albumin ratio was set at 3.04 based on the ROC analysis. Multivariate analysis identified C-reactive protein/albumin ratio was an independent risk factors for complications after gastrectomy $(\mathrm{OR} 3.037 ; 95 \% \mathrm{Cl}$ 1.248-7.392; $P=0.014)$. Additionally, $C$-reactive protein/albumin ratio showed a higher diagnostic accuracy than C-reactive protein on postoperative day 3 (AUC: 0.685 vs 0.660; sensitivity: 0.624 vs 0.471 ; specificity: 0.722 vs 0.835$)$.

Conclusions: Elevated C-reactive protein/albumin ratio was an independent predictor for postoperative complications following gastrectomy of gastric cancer, and the diagnostic accuracy was higher than C-reactive protein alone. Overall, postoperative C-reactive protein/albumin ratio may help to identify patients with high probability of postoperative complications.
\end{abstract}

Keywords: C-reactive protein to albumin ratio, Postoperative complications, Gastric cancer

\section{Background}

There are almost 1,000,000 new cases of gastric cancer every year worldwide, and half of these occur in Eastern Asia, particularly in China. Although the incidence of gastric cancer has declined over the years, it remains the fifth most common cancer and the third leading cause of cancer-related death in the world [1]. So far, surgical resection is still the primary treatment for resectable gastric cancer. Concomitantly, gastrectomy for gastric

\footnotetext{
* Correspondence: medguanwenxian@163.com

${ }^{1}$ Department of General Surgery, Drum Tower Hospital, Medical School of

Nanjing University, Nanjing, Jiangsu 210008, China

Full list of author information is available at the end of the article
}

cancer may lead to high rates of postoperative complication, which has a negative effect on hospital recovery and long-term survival [2-5]. So, it is necessary to identify accurate predictive factors to predict postoperative complications early after surgery.

Several systemic inflammatory markers, including the Glasgow Prognostic Score (GPS), C-reactive protein (CRP), platelet to lymphocyte ratio (PLR), and neutrophil to lymphocyte ratio (NLR), have been established to predict postoperative complication [6]. As an acutephase protein, CRP was widely studied in large number of surgery $[7,8]$. Recently, a few studies began studying the predictive value of CRP for gastric cancer resection 
[9, 10]. In addition, predictors showing nutritional status, such as hypoalbuminemia, low body mass index (BMI), and weight loss, were also reported to be associated with postoperative recovery of gastric cancer $[11,12]$.

As a combination of these two aspects, C-reactive protein/albumin ratio (CAR) has been shown to be a promising prognostic index in pancreatic cancer [13], colorectal cancer, and renal cell cancer et al. [14, 15]. Liu et al. and Toiyama et al. have reported, respectively, that elevated CAR was related to a poor prognosis for gastric cancer resection [16, 17]. The former studies have largely been focused on preoperative CAR but to a lesser extent on that after surgery. Until now, whether altered postoperative CAR is associated with poor prognosis remains unclear. In this study, we evaluated the predictive value of postoperative CAR for short-term complications after gastric cancer resection.

\section{Methods}

\section{Patients}

A total of 322 patients who underwent curative (R0) gastrectomy between October 2015 and March 2017 in Nanjing Drum Tower Hospital were retrospectively analyzed. All of the patients were histologically confirmed, and blood laboratory tests on postoperative day (POD) 3 were complete. The exclusion criteria were as follows: (1) patients accepting ALB infusion preoperatively or within POD 3; (2) patients with liver cirrhosis and infection before gastrectomy which may have an influence on the serum albumin level; (3) reoperation within POD 3; (4) multivisceral resection. This study was approved by the Ethics Committee of Drum Tower Hospital, Medial School of Nanjing University.

\section{Data extraction}

Data extraction included three aspects: the baseline characteristics, laboratory tests, and intraoperative index. The baseline characteristics were collected, including age, gender, BMI, comorbidities, and American Society of Anesthesiologists (ASA) grade. Blood laboratory tests included preoperative serum albumin, hemoglobin, CRP; postoperative CRP, albumin on POD 3; tumor markers [carbohydrate antigen 19-9 (CA19-9), carcinoembryonic antigen (CEA)]. Intraoperative index involved operation time, surgical approach, degree of lymph node dissection, and blood loss. Tumor stage was based on the 7th Edition of TNM Classification for Gastric Cancer [18].

\section{Definition of postoperative complications}

The postoperative complications were termed as those occurred in hospital or within 30 days after surgery. All complications were categorized based on the ClavienDindo classification system [19]. Patients with postoperative complications of grades I or II were divided into minor group, while patients with complications of grades III or more were divided into major group. Besides postoperative short-term complications, the length of hospital stay was also collected to evaluate outcomes of patients with surgery. The CRP/Alb ratio was calculated as serum CRP level to serum ALB level both on POD 3 $[14,20]$. CRP and CAR cutoff threshold were both determined based on receiver operating characteristic (ROC) curve analysis.

\section{Statistical analysis}

Continuous variable data were presented as means $\pm \mathrm{SE}$ and analyzed using Student's $t$ test or Mann-Whitney $U$ test. Categorical variable data were presented as number (\%) and analyzed using the Chi squared test or the fisher exact test. Univariate and multivariate analyses were performed to evaluate risk factors for the early postoperative complications. Accuracy of each optional risk factor was measured using receiver operating characteristic analysis. All of the statistical analyses were performed using SPSS 19.0 (Chicago, IL, USA), and statistical differences were termed as $P<0.05$.

\section{Results}

\section{Patient characteristics}

Of the total of 322 patients, 232 were male while 90 were female. Among these patients with gastric cancer, 300 (93.2\%) underwent open gastrectomy and 22 (6.8\%) underwent laparoscopic surgery. Total gastrectomy was performed in $170(52.8 \%)$ patients; distal gastrectomy and proximal gastrectomy were, respectively, in 114 (35.4\%) and $38(11.8 \%)$. According to the 7th Edition of TNM Classification for Gastric Cancer, the number of patients with stage I/II/III/IV were 101/58/148/15 respectively. The mean operation time was $236.7 \pm 65.7 \mathrm{~min}$; mean blood loss was $229.6 \pm 145.5 \mathrm{ml}$; mean length of postoperative stay was $15.4 \pm 7.8$ days. Overall, 85 (26.4\%) patients had postoperative complications. Of those, $56(17.4 \%)$ patients had minor complications $\left[10\right.$ fever $\left(>38.5{ }^{\circ} \mathrm{C}\right)$ after surgery, 5 dumping syndrome, 17 delayed gastric emptying, 2 intestinal pseudoobstruction, 11 wound infection, 3 anemia and 8 TPN $>2$ weeks], while $29(9.0 \%)$ had major complications (5 anastomotic leakage, 5 fascial dehiscence, 2 adhesive intestinal obstruction, 3 abdominopelvic collection, 8 pleural effusion, 3 lymphatic leakage, 2 intra-abdominal bleeding and 1 death) according to the Clavien-Dindo classification system. Detailed clinicopathologic characteristics of all patients were shown in Table 1.

\section{Association between clinicopathologic characteristics and postoperative complications}

As shown in Table 2, univariate analysis revealed that postoperative complications were significantly associated 
Table 1 Demographic and clinical features of patients

\begin{tabular}{|c|c|c|c|}
\hline Characteristic & $N=322$ & Characteristic & $N=322$ \\
\hline Age (years) & $62.3 \pm 9.9$ & CRP on POD 3 (mg/L) & $96.7 \pm 59.5$ \\
\hline Gender $(n)$ & & ALB on POD $3(\mathrm{~g} / \mathrm{L})$ & $32.9 \pm 3.6$ \\
\hline Male & 232 & $A S A \geq 3$ & 171 \\
\hline Female & 90 & Clinical stage I/II/II//V & $101 / 58 / 148 / 15$ \\
\hline BMI $\left(\mathrm{kg} / \mathrm{m}^{2}\right)$ & $23.0 \pm 3.3$ & Mode of surgical approach $(n, \%)$ & \\
\hline Comorbidities ( $n$ ) & & Laparoscopic & 22 \\
\hline Diabetes mellitus & 20 & Open & 300 \\
\hline Hypertension & 111 & Type of resection $(n, \%)$ & \\
\hline Preoperative serum albumin ( $g / L)$ & $38.6 \pm 3.2$ & Distal gastrectomy & 114 \\
\hline Preoperative hemoglobin ( $\mathrm{g} / \mathrm{L}$ ) & $123.1 \pm 25.2$ & Proximal gastrectomy & 38 \\
\hline Preoperative CRP (g/L) & $6.2 \pm 10.4$ & Total gastrectomy & 170 \\
\hline CA 19-9 (ng/ml) & & Degree of lymph node dissection (D) $\geq 2$ & 246 \\
\hline$\geq 37$ & 60 & Operation time (min) & $236.7 \pm 65.7$ \\
\hline$<37$ & 262 & Blood loss (ml) & $229.6 \pm 145.5$ \\
\hline CEA (ng/ml) & & Postoperative complications (Clavien-Dindo) & \\
\hline$\geq 5$ & 39 & Grades I and II & 56 \\
\hline$<5$ & 283 & $\geq$ Grade III & 29 \\
\hline Lymphocyte count $\left(\times 10^{9} / \mathrm{L}\right)$ & & Postoperative stay (days) & $15.4 \pm 7.8$ \\
\hline$\geq 3$ & 13 & & \\
\hline$<3$ & 309 & & \\
\hline
\end{tabular}

BMI body mass index, CA 19-9 carbohydrate antigen 19-9, CEA carcinoembryonic antigen, CRP C-reactive protein, $P O D$ postoperative day, ASA American Society of Anesthesiologists, $A L B$ albumin

with CRP on POD 3, postoperative CAR, and degree of lymph node dissection. In further multivariate analysis, unlike CRP on POD 3, postoperative CAR (OR 3.037; 95\% CI 1.248-7.392; $P=0.014$ ) was still significantly associated with postoperative complications. These data indicated that postoperative CAP might be an independent predictor for early postoperative complications.

\section{Predictive value of CAR for postoperative complications compared with CRP on POD 3}

Previous work demonstrated that postoperative CRP could be an optional predictor for complications after gastrectomy $[9,10]$. To compare the predictive accuracy of postoperative CRP and postoperative CAR, receiver operating characteristic curve was performed. According to ROC analysis of all complications, the optimal cut-off value was 131.9 for CRP on POD 3, 3.04 for CAR respectively. ROC curve parameters were shown in Fig. 1. The AUC of CRP on POD 3 was 0.660 , sensitivity was 0.471 , specificity was 0.835 , and Youden's index was 0.306 . In contrast, the AUC of CAR was 0.685 , sensitivity was 0.624 , specificity was 0.722 , and Youden's index was 0.345 . All these data showed that postoperative CAR might be a better predictor for early postoperative complications than postoperative CRP.

\section{Postoperative CAR as a predictor for postoperative complications}

Patients were divided into two groups: high CAR $(\geq$ 3.04), low CAR (<3.04). Patients with high CAR were more likely to have postoperative complications than those with low CAR ( 44.5 vs $15.8 \%, P<0.001$ ). Specifically, the possibility of suffering mild complications (grades I and II) and major complications (grades III or more) in patients with high CAR were both higher than those with low CAR (30.3 vs $9.9 \%, p<0.001 ; 14.3$ vs $5.9 \%, P=0.011)$. In addition, patients with CAR also had prolonged hospital stay $(17.1 \pm 10.3$ vs $14.5 \pm 5.6$, $P=0.003$ ) (Table 3).

\section{Discussion}

In this study, patients with high postoperative CAR were more likely to have postoperative complications, and prolonged hospital stay. Additionally, postoperative CAR seemed to be more accurate to predict complications than postoperative CRP. Therefore, the postoperative CAR might be a promising predictor for postoperative complications after gastrectomy for gastric cancer.

Surgical resection is the main treatment for resectable gastric cancer. Although gastric cancer resection greatly prolongs the survival of patients, it brings some harmful effects. The overall morbidity of postoperative complications 
Table 2 Univariate and multivariate analyses of risk factors associated with postoperative complications

\begin{tabular}{|c|c|c|c|c|c|c|}
\hline \multirow[b]{2}{*}{ Characteristics } & \multicolumn{3}{|c|}{ Univariate } & \multicolumn{3}{|c|}{ Multivariate } \\
\hline & OR & $95 \% \mathrm{Cl}$ & $P$ & OR & $95 \% \mathrm{Cl}$ & $P$ \\
\hline Age ( $\geq 75$ years) & 1.441 & $0.621-3.343$ & 0.395 & 1.827 & $0.641-5.212$ & 0.260 \\
\hline Sex & 0.721 & $0.422-1.234$ & 0.233 & 0.703 & $0.361-1.371$ & 0.301 \\
\hline BMI $(<18.5$ kg/m²) & 0.384 & $0.085-1.725$ & 0.212 & 0.269 & $0.050-1.447$ & 0.126 \\
\hline \multicolumn{7}{|l|}{ Comorbidities } \\
\hline Diabetes mellitus & 2.433 & $0.971-6.096$ & 0.058 & 2.310 & $0.784-6.812$ & 0.129 \\
\hline Hypertension & 0.628 & $0.364-1.085$ & 0.095 & 0.717 & $0.364-1.411$ & 0.335 \\
\hline Preoperative serum albumin $(<35 \mathrm{~g} / \mathrm{L})$ & 0.745 & $0.326-1.701$ & 0.485 & 0.274 & $0.092-0.810$ & 0.019 \\
\hline Preoperative hemoglobin (<120 g/L) & 1.587 & $0.957-2.630$ & 0.073 & 2.023 & $1.027-3.985$ & 0.042 \\
\hline Preoperative CRP ( $\geq 10 \mathrm{~g} / \mathrm{L}$ ) & 1.030 & $0.417-2.544$ & 0.949 & 0.965 & $0.304-3.059$ & 0.951 \\
\hline CA 19-9 ( $\geq 37$ ng/ml) & 1.376 & $0.747-2.536$ & 0.306 & 1.363 & $0.615-3.020$ & 0.446 \\
\hline CEA ( $\geq 5 \mathrm{ng} / \mathrm{ml}$ ) & 0.956 & $0.445-2.057$ & 0.909 & 0.681 & $0.267-1.738$ & 0.422 \\
\hline Lymphocyte count ( $\left.\geq 3 \times 10^{9} / L\right)$ & 0.223 & $0.029-1.743$ & 0.153 & 0.164 & $0.019-1.447$ & 0.104 \\
\hline CRP on POD 3 (mg/L) & 4.513 & $2.611-7.799$ & $<0.001$ & 2.376 & $0.955-5.913$ & 0.063 \\
\hline Postoperative CAR & 4.291 & $2.544-7.237$ & $<0.001$ & 3.037 & $1.248-7.392$ & 0.014 \\
\hline$A S A \geq 3$ & 0.818 & $0.498-1.343$ & 0.427 & 0.793 & $0.415-1.519$ & 0.485 \\
\hline Clinical stage ( $\geq I I)$ & 1.010 & $0.590-1.729$ & 0.971 & 0.565 & $0.271-1.177$ & 0.128 \\
\hline Mode of surgical approach & 0.809 & $0.289-2.264$ & 0.686 & 0.787 & $0.228-2.715$ & 0.704 \\
\hline Type of resection & 0.945 & $0.576-1.553$ & 0.824 & 0.858 & $0.452-1.630$ & 0.641 \\
\hline Degree of lymph node dissection $(D) \geq 2$ & 2.542 & $1.269-5.092$ & 0.008 & 4.429 & $1.884-10.410$ & 0.001 \\
\hline Operation time ( $\geq 250 \mathrm{~min}$ ) & 1.544 & $0.934-2.553$ & 0.090 & 1.513 & $0.791-2.893$ & 0.211 \\
\hline Blood loss ( $\geq 200 \mathrm{ml}$ ) & 1.443 & $0.845-2.466$ & 0.179 & 1.424 & $0.762-2.659$ & 0.268 \\
\hline
\end{tabular}

BMI body mass index, CA 19-9 carbohydrate antigen 19-9, CEA Carcinoembryonic antigen, CRP C-reactive protein, POD Postoperative day, ASA American Society of Anesthesiologists, CAR CRP to albumin (CRP/ALB) ratio, OR odds ratio, Cl confidence interval

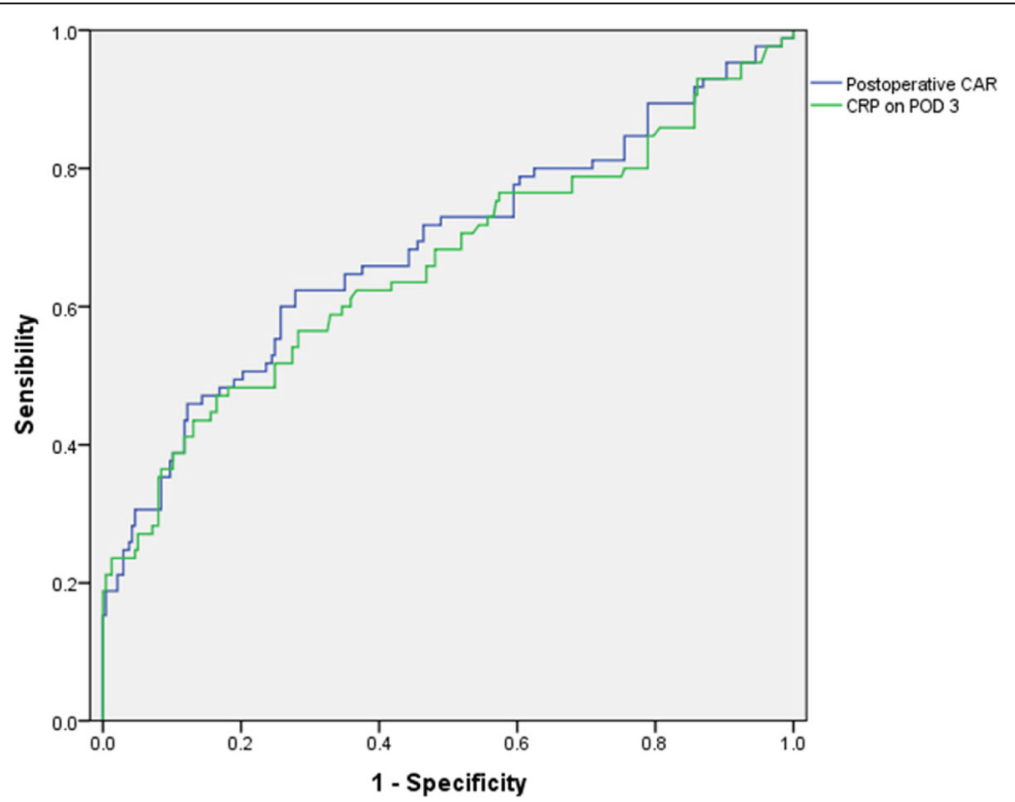

Fig. 1 Receiver operating characteristic curve showing postoperative CAR and C-reactive protein on postoperative day 3 levels predictive of postoperative overall complications 
Table 3 Comparison of postoperative complications associated with postoperative CAR

\begin{tabular}{lllll}
\hline Characteristics & All $(n=322)$ & CAR $<3.04(n=203)$ & CAR $\geq 3.04(n=119)$ & $P 3(44.5 \%)$ \\
\hline Overall, $n(\%)^{a, c}$ & $85(26.4)$ & $32(15.8 \%)$ & $36(30.3 \%)$ & $<0.001$ \\
Grades I or II, $n(\%)^{a, c}$ & $56(17.4)$ & $20(9.9 \%)$ & $17(14.3 \%)$ & $<0.001$ \\
Grade III or greater, $n(\%)^{a, c}$ & $29(9.0)$ & $12(5.9 \%)$ & $17.1 \pm 10.3$ & 0.011 \\
Postoperative stay (days) & $15.4 \pm 7.8$ & $14.5 \pm 5.6$ & 0.003 \\
\hline
\end{tabular}

CAR C-reactive protein to albumin ratio

${ }^{a}$ Clavien-Dindo's classification of surgical complication

${ }^{b}$ Values are expressed as the mean \pm SD

'Values are expressed as $\mathrm{n}(\%)$

and mortality rates were reported to be 13 to 38 and 2 to $8.5 \%$, respectively [21]. Postoperative complications, such as anastomotic leakage, and abdominal abscess have a negative effect on short-term surgical outcomes. Patients would have higher medical costs, and prolonged hospital stays. On the other hand, postoperative complications, especially infection complications, could cause prolonged inflammation which provides an appropriate microenvironment for recurrence of gastric cancer, and finally affect long-term survival [2, 5, 22]. Thus, no matter in terms of short-term or long-term outcomes, postoperative complications can bring catastrophic effects. So, it makes sense to find an accurate biochemical marker to predict postoperative complications in early stage.

Previous studies have proven that either preoperative or postoperative CRP could be an important predictive factor for both short-term outcomes and long-term mortality [10, 23-25]. As one of the inflammatory markers, CRP elevation mainly owes to the inflammatory reaction for cancer and surgical procedure. Shishido and colleagues evaluated that postoperative CRP on POD 3 could predict infectious complications after gastric cancer resection [10]. Kim et al. also demonstrated that postoperative CRP was a more accurate predictor for postoperative complications than other inflammatory markers, such as platelet count, neutrophil count, and ratios of these two factors [9]. Although postoperative CRP holds promise for prediction postoperative complications, there are still some limitations for being used widely in clinical practice. The main drawbacks of CRP are predictive accuracy and time lag [26, 27]. A recent study showed that the time point postoperative CRP began changing was late than some other inflammatory markers, like Interleukin-6 [26]. In our study, multivariate analysis also showed that postoperative CRP on POD 3 was not an independent risk factor for complications following gastrectomy.

To improve the accuracy of CRP, we modified this predictive index by introducing another factor: albumin. Albumin is produced in liver and accounts for the most abundant serum protein [28]. The serum albumin declines in patients with poor nutritional status, loss of skeletal muscle, and systemic inflammatory response [29]. The decline in albumin is due to the reprioritizes from visceral proteins to acute phase proteins happening in the liver, capillary leakage and hemodilution with fluid infusion [30, 31]. Furthermore, hypoalbuminemia has been proven to be a predictive marker for postoperative outcomes [32]. Ryan et al. revealed that postoperative hypoalbuminemia was associated with complications after esophagectomy [33]. Ge and his colleague also showed that the decrease of serum albumin could predict postoperative complications following colorectal resection [34]. In this study, we merged CRP and albumin together to calculate a single index: CRP/Alb ratio. Evaluation showed that postoperative CAR had higher AUC than postoperative CRP alone, and was an independent risk factor for postoperative complications following gastrectomy.

In this article, we chose the POD 3 as the time point for calculating CAR for several reasons. On one hand, there have been compelling suggestions that postoperative CRP reaches a peak at POD 3 or 4 , and its predictive accuracy is better than that in POD 1 or $2[9,10]$. On the other hand, the minimal albumin levels begin in 4-6 h after surgery and last for 3 days [27, 35]. Since both CRP and albumin own the maximal amplitude on POD 3, it would be reasonable to assume that CAR on POD 3 has the highest predictive accuracy.

To our knowledge, this is the first study to consider the postoperative CAR as a predictor for postoperative complications following gastrectomy for gastric cancer. Our results corresponded to previous studies in other kinds of cancer, such as hepatocellular cancer [36], lung cancer [37], and pancreatic cancer [13] et al. Although a good many research groups studied CAR, most of them focused on preoperative CAR. Considering inflammation from surgical trauma, postoperative CAR may be also a promising predictor for postoperative complications. Taken together, early detection of postoperative CAR may be beneficial to take action promptly before critical complications develop.

We acknowledge that there are some limitations in this study. First, the use of CAR on POD 3 may be a bit late for surgeons to perform preventive interventions. 
Second, it was a retrospective study, so it may be flawed by residual confounding factors. Third, patients enrolled in this study were from a single center. As the surgical technique and perioperative management played an important effect on postoperative complications, multicenter studies are needed to confirm our results. Lastly, the CAR cut-off value may be biased in this study for it was calculated using ROC analysis.

\section{Conclusions}

In conclusion, the current study demonstrated that postoperative CAR is an independent predictive marker for short-term complications following gastrectomy of gastric cancer. Patients with high CAR $(\geq 3.04)$ need to be treated seriously before critical complications develop.

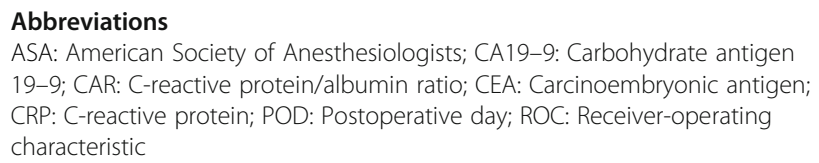

\section{Abbreviations}

ASA: American Society of Anesthesiologists; CA19-9: Carbohydrate antigen 19-9; CAR: C-reactive protein/albumin ratio; CEA: Carcinoembryonic antigen; CRP: C-reactive protein; POD: Postoperative day; ROC: Receiver-operating characteristic

\section{Acknowledgements}

The authors gratefully acknowledge all of the investigators for their contributions to the trial.

\section{Availability of data and materials}

This work was supported by the National Natural Science Foundation of China (No. 81372364) and the State Key Program of Nanjing, China (No. ZKX 14022). Access to the data and the calculation method can be obtained from the authors by email (medsunfeng@163.com).

\section{Authors' contributions}

FS contributed to the study design and drafted the manuscript. XG worked on the study design and data analysis. ZL was involved in the data collection and extraction. SD and SA collected the data. WG revised the manuscript. All authors have read and approved the final manuscript.

\section{Ethics approval and consent to participate}

This study was approved by the ethics committee of Nanjing Drum Tower Hospital, Medial School of Nanjing University. Written informed consent was obtained from all participants.

\section{Consent for publication}

Written informed consent was obtained from all patients enrolled in the investigation. The study protocol conformed to the guidelines of the regional ethical committees of Nanjing Drum Tower Hospital, Medial School of Nanjing University.

\section{Competing interests}

The authors declare that they have no competing interests.

\section{Publisher's Note}

Springer Nature remains neutral with regard to jurisdictional claims in published maps and institutional affiliations.

\section{Author details}

'Department of General Surgery, Drum Tower Hospital, Medical School of Nanjing University, Nanjing, Jiangsu 210008, China. ²Department of General Surgery, Sir Run Run Shaw Hospital, School of Medicine, Zhejiang University, Hangzhou, Zhejiang 310016, China.
Received: 30 August 2017 Accepted: 15 October 2017

Published online: 24 October 2017

\section{References}

1. Stewart B, Wild CP. World cancer report 2014: World; 2014. http:// publications.iarc.fr/Non-Series-Publications/World-Cancer-Reports/WorldCancer-Report-2014

2. Tsujimoto $H$, Ichikura $T$, Ono $S$, Sugasawa $H$, Hiraki S, Sakamoto $N$, Yaguchi Y, Yoshida K, Matsumoto Y, Hase K. Impact of postoperative infection on long-term survival after potentially curative resection for gastric cancer. Ann Surg Oncol. 2009;16(2):311-8.

3. Yoo HM, Lee HH, Shim JH, Jeon HM, Park CH, Song KY. Negative impact of leakage on survival of patients undergoing curative resection for advanced gastric cancer. J Surg Oncol. 2011;104(7):734-40. doi: 10.1002/jso.22045.

4. Tokunaga M, Tanizawa Y, Bando E, Kawamura T, Terashima M. Poor survival rate in patients with postoperative intra-abdominal infectious complications following curative gastrectomy for gastric cancer. Ann Surg Oncol. 2013; 20(5):1575-83. doi: 10.1245/s10434-012-2720-9.

5. Kubota T, Hiki N, Sano T, Nomura S, Nunobe S, Kumagai K, Aikou S, Watanabe $\mathrm{R}$, Kosuga T, Yamaguchi T. Prognostic significance of complications after curative surgery for gastric cancer. Ann Surg Oncol. 2014;21(3):891-8.

6. Moyes LH, Leitch EF, McKee RF, Anderson JH, Horgan PG, McMillan DC. Preoperative systemic inflammation predicts postoperative infectious complications in patients undergoing curative resection for colorectal cancer. Br J Cancer. 2009;100(8):1236-9. doi: 10.1038/sj.bjc.6604997.

7. Shimizu T, Ishizuka M, Kubota K. The preoperative serum C-reactive protein level is a useful predictor of surgical site infections in patients undergoing appendectomy. Surg Today. 2015;45(11):1404-10. doi: 10.1007/s00595-014-1086-y.

8. Selby J, Prabhudesai A. Can C-reactive protein predict the severity of a postoperative complication after elective resection of colorectal cancer? Int J Color Dis. 2014;29(10):1211-5. doi: 10.1007/s00384-014-1977-9.

9. Kim EY, Yim HW, Park CH, Song KY. C-reactive protein can be an early predictor of postoperative complications after gastrectomy for gastric cancer. Surg Endosc Interv Tech. 2017;31(1):445-54. doi: 10.1007/s00464-016-5272-4.

10. Shishido Y, Fujitani K, Yamamoto K, Hirao M, Tsujinaka T, Sekimoto M. Creactive protein on postoperative day 3 as a predictor of infectious complications following gastric cancer resection. Gastric Cancer. 2016;19(1): 293-301. doi: 10.1007/s10120-014-0455-y.

11. Toiyama Y, Yasuda H, Ohi M, Yoshiyama S, Araki T, Tanaka K, Inoue Y, Mohri $Y$, Kusunoki M. Clinical impact of preoperative albumin to globulin ratio in gastric cancer patients with curative intent. Am J Sur. 2017;213(1):120-6. doi: 10.1016/j.amjsurg.2016.05.012.

12. Liu X, Qiu H, Kong P, Zhou Z, Sun X. Gastric cancer, nutritional status, and outcome. Oncotargets Ther. 2017;10:2107-14. doi: 10.2147/ott.s132432.

13. Wu M, Guo J, Guo L, Zuo Q. The C-reactive protein/albumin ratio predicts overall survival of patients with advanced pancreatic cancer. Tumor Biol. 2016;37(9):12525-33.

14. Ge X, Cao Y, Wang H, Ding C, Tian H, Zhang X, Gong J, Zhu W, Li N. Diagnostic accuracy of the postoperative ratio of $C$-reactive protein to albumin for complications after colorectal surgery. World J Surg Oncol. 2017;15 doi: 10.1186/s12957-016-1092-1.

15. Guo S, He X, Chen Q, Yang G, Yao K, Dong P, Ye Y, Chen D, Zhang Z, Qin Z, Liu Z, Xue Y, Zhang M, Liu R, Zhou F, Han H. The C-reactive protein/albumin ratio, a validated prognostic score, predicts outcome of surgical renal cell carcinoma patients. Bmc Cancer 17. 2017; doi: 10.1186/s12885-017-3119-6.

16. Liu X, Sun X, Liu J, Kong P, Chen S, Zhan Y, Xu D. Preoperative C-reactive protein/albumin ratio predicts prognosis of patients after curative resection for gastric cancer. Transl Oncol. 2015;8(4):339-45. doi: 10.1016/j.tranon.2015.06.006.

17. Toiyama $Y$, Shimura $T$, Yasuda $H$, Fujikawa $H$, Okita $Y$, Kobayashi M, Ohi M, Yoshiyama S, Hiro J, Araki T, Inoue Y, Mohri Y, Kusunoki M. Clinical burden of C-reactive protein/albumin ratio before curative surgery for patients with gastric cancer. Anticancer Res. 2016;36(12):6491-8. doi: 10.21873/anticanres.11248.

18. Edge SB, Compton CC. The American Joint Committee on Cancer: the 7th edition of the AJCC cancer staging manual and the future of TNM. Ann Surg Oncol. 2010;17(6):1471-4. doi: 10.1245/s10434-010-0985-4

19. Clavien PA, Barkun J, de Oliveira ML, Vauthey JN, Dindo D, Schulick RD, de Santibanes E, Pekolj J, Slankamenac K, Bassi C, Graf R, Vonlanthen R, Padbury $\mathrm{R}$, Cameron JL, Makuuchi M. The Clavien-Dindo classification of surgical 
complications five-year experience. Ann Surg. 2009;250(2):187-96. doi: 10.1097/SLA.0b013e3181b13ca2.

20. Ranzani OT, Zampieri FG, Forte DN, Pontes Azevedo LC, Park M. C-reactive protein/albumin ratio predicts 90-day mortality of septic patients. PLoS One. 2013;8(3) doi: 10.1371/journal.pone.0059321.

21. Bartlett EK, Roses RE, Kelz RR, Drebin JA, Fraker DL, Karakousis GC. Morbidity and mortality after total gastrectomy for gastric malignancy using the American College of Surgeons National Surgical Quality Improvement Program database. Surgery. 2014;156(2):298-304.

22. Hayashi T, Yoshikawa T, Aoyama T, Hasegawa S, Yamada T, Tsuchida K, Fujikawa H, Sato T, Ogata T, Cho H. Impact of infectious complications on gastric cancer recurrence. Gastric Cancer. 2015;18(2):368-74.

23. Kong F, Gao F, Chen J, Zheng R, Liu H, Li X, Yang P, Liu G, Jia Y. Elevated serum C-reactive protein level predicts a poor prognosis for recurrent gastric cancer. Oncotarget. 2016;7(34):55765-70. doi: 10.18632/oncotarget.9910.

24. Zhang K, Xi H, Wu X, Cui J, Bian S, Ma L, Li J, Wang N, Wei B, Chen L. Ability of serum C-reactive protein concentrations to predict complications after laparoscopy-assisted Gastrectomy: a prospective cohort study. Medicine. 2016;95(21) doi: 10.1097/md.0000000000003798.

25. Welsch T, Frommhold K, Hinz U, Weigand MA, Kleeff J, Friess H, Buechler MW, Schmidt J. Persisting elevation of C-reactive protein after pancreatic resections can indicate developing inflammatory complications. Surgery. 2008;143(1):20-8. doi: 10.1016/j.surg.2007.06.010.

26. Rettig TCD, Verwijmeren L, Dijkstra IM, Boerma D, Van De Garde EMW Noordzij PG. Postoperative Interleukin-6 level and early detection of complications after elective major abdominal surgery. Ann Surg. 2016; 263(6):1207-12. doi: 10.1097/sla.0000000000001342.

27. Hubner M, Mantziari S, Demartines N, Pralong F, Coti-Bertrand P, Schafer M. Postoperative albumin drop is a marker for surgical stress and a predictor for clinical outcome: a pilot study. Gastroenterol Res Pract. 2016;8 doi: 10.1155/2016/8743187.

28. Fuhrman MP. The albumin-nutrition connection: separating myth from fact. Nutrition. 2002;18(2):199-200. doi: 10.1016/s0899-9007(01)00729-8.

29. Gabay C, Kushner I. Acute-phase proteins and other systemic responses to inflammation. N Engl J Med. 1999:340(6):448-54.

30. Soeters P, Von Meyenfeldt M, Meijerink WH, Fredrix EH, Wouters EM, Schols AW, Williams W, Pawlowicz A, Davies B, Geril MDL (1990) Serum albumin and mortality. Lancet 335 (8685):348-351.

31. Lee WL, Slutsky AS. Sepsis and endothelial permeability. N Engl J Med. 2010;363(7):689.

32. Moghadamyeghaneh Z, Hwang G, Hanna MH, Phelan MJ, Carmichael JC, Mills SD, Pigazzi A, Dolich MO, Stamos MJ. Even modest hypoalbuminemia affects outcomes of colorectal surgery patients. Am J Surg. 2015;210(2):276-84. doi: 10.1016/j.amjsurg.2014.12.038.

33. Ryan AM, Hearty A, Prichard RS, Cunningham A, Rowley SP, Reynolds JV. Association of hypoalbuminemia on the first postoperative day and complications following esophagectomy. J Gastrointest Surg. 2007:11(10):1355-60. doi: 10.1007/s11605-007-0223-y.

34. Ge X, Dai X, Ding C, Tian H, Yang J, Gong J, Zhu W, Li N, Li J. Early postoperative decrease of serum albumin predicts surgical outcome in patients undergoing colorectal resection. Dis Colon Rectum. 2017;60(3):326-34. doi: $10.1097 /$ dcr.0000000000000750

35. Fleck A, Hawker F, Wallace P, Raines G, Trotter J, Ledingham IM, Calman K. Increased vascular permeability: a major cause of hypoalbuminaemia in disease and injury. Lancet. 1985;325(8432):781-4.

36. Kinoshita A, Onoda H, Imai N, Iwaku A, Oishi M, Tanaka K, Fushiya N, Koike $\mathrm{K}$, Nishino $\mathrm{H}$, Matsushima M. The C-reactive protein/albumin ratio, a novel inflammation-based prognostic score, predicts outcomes in patients with hepatocellular carcinoma. Ann Surg Oncol. 2015;22(3):803-10.

37. Zhou T, Zhan J, Hong S, Hu Z, Fang W, Qin T, Ma Y, Yang Y, He X, Zhao Y. Ratio of C-reactive protein/albumin is an inflammatory prognostic score for predicting overall survival of patients with small-cell lung cancer. Sci Rep. 2015;5:10481.

\section{Submit your next manuscript to BioMed Central and we will help you at every step:}

- We accept pre-submission inquiries

- Our selector tool helps you to find the most relevant journal

- We provide round the clock customer support

- Convenient online submission

- Thorough peer review

- Inclusion in PubMed and all major indexing services

- Maximum visibility for your research

Submit your manuscript at www.biomedcentral.com/submit

) Biomed Central 